\title{
MULTILINEAR ESTIMATES AND FRACTIONAL INTEGRATION
}

\author{
Carlos E. Kenig and Elias M. Stein
}

\section{Introduction}

The purpose of this note is to describe several results about multilinear operators of fractional integral type. The simplest example that arises is the bilinear operator acting on functions of $\mathbb{R}^{n}$ given by

$$
I(f, g)(x)=\int_{\mathbb{R}^{n}} f(x-y) g(x+y) K(y) d y,
$$

where $K(y)=|y|^{-n+\alpha}, 0<\alpha<n$.

Now if $n=1$, and we take instead of the locally integrable kernel $K(y)=$ $|y|^{-1+\alpha}$ the singular integral kernel $K(y)=1 / y$, then $I$ becomes the bilinear Hilbert transform. In that case, we know by the work of Lacey and Thiele [L-T] that $I$ maps $L^{p_{1}} \times L^{p_{2}} \rightarrow L^{r}$, where

$$
\frac{1}{r}=\frac{1}{p_{1}}+\frac{1}{p_{2}}, \quad 1<p_{2}<\infty,
$$

and the $p_{i}$ are additionally restricted by the requirement $r>\frac{2}{3}$ (instead of $r>\frac{1}{2}$ ). The condition $r>\frac{2}{3}$ is a consequence of the methods used in their proof, and may not be necessary; these methods are intricate and at a crucial point rely on $L^{2}$ estimates.

Returning to the fractional integration case $K(y)=|y|^{-n+\alpha}$, we shall prove below - by very elementary considerations - that $I$ maps $L^{p_{1}} \times L^{p_{2}} \rightarrow L^{r}$ for the full range: i.e., when $1<p_{i} \leq \infty, i=1,2$, and

$$
\frac{1}{r}=\frac{1}{p_{1}}+\frac{1}{p_{2}}-\frac{\alpha}{n}
$$

with the only other limitation being $0<\alpha$, and $r<\infty$. (These last two restrictions are necessary for the integral $I$ to be finite; i.e., for all $f, g \in L^{p_{1}} \times$ $L^{p_{2}}$.)

Received October 29, 1998.

Both authors were supported in part by the NSF. 
We will establish this result in the greater generality of $(k+1)$-linear mappings, with $k \geq 1$. Thus we shall consider an operator $I_{\alpha}$ defined by

$$
\begin{aligned}
& I_{\alpha}\left(f_{1}, f_{2}, \ldots, f_{k+1}\right)(x) \\
& \quad=\int_{\mathbb{R}^{n k}} f_{1}\left(\ell_{1}\right) f_{2}\left(\ell_{2}\right) \cdots f_{k+1}\left(\ell_{k+1}\right) K\left(x_{1}, \ldots, x_{k}\right) d x_{1} \cdots d x_{k} .
\end{aligned}
$$

Here $x_{j} \in \mathbb{R}^{n}, 1 \leq j \leq k$ and also $x \in \mathbb{R}^{n}$; so $\left(x_{1}, \ldots, x_{k}\right) \in \mathbb{R}^{n k}$ and $\left(x_{1}, \ldots, x_{k}, x\right) \in \mathbb{R}^{n(k+1)}$. The $\ell_{j}=\ell_{j}\left(x_{1}, \ldots, x_{k}, x\right)$, for $1 \leq j \leq k+1$, are linear mappings from $\mathbb{R}^{n(k+1)}$ to $\mathbb{R}^{n}$, which are assumed to be appropriately non-degenerate and independent (see assumptions (H1), (H2) and (H3) below). If we set

$$
K\left(x_{1}, \ldots, x_{k}\right)=\left|\left(x_{1}, \ldots, x_{k}\right)\right|^{-n k+\alpha},
$$

then we obtain the boundedness of $I_{\alpha}$ from $L^{p_{1}} \times L^{p_{2}} \times \cdots \times L^{p_{k+1}}$ to $L^{r}$ when

$$
\frac{1}{r}=\sum_{j=1}^{k+1} \frac{1}{p_{j}}-\frac{\alpha}{n},
$$

under the assumptions that $1<p_{j} \leq \infty$ for all $j$, and $\alpha>0, r<\infty$. If under the same circumstances we relax the restrictions on the $p_{j}$ so that $1 \leq p_{j} \leq \infty$, then the mapping is bounded from $L^{p_{1}} \times \cdots \times L^{p_{k+1}}$ to weak $L^{r}$. In the case when $r \geq 1$, our results can be obtained using the rearrangement inequality in [B-L]. However, this approach does not apply to the more novel case $r<1$.

The multilinear results above raise naturally several additional questions.

(1) For multilinear operators of the above kind, with integration over $\mathbb{R}^{n k}$, what happens if they involve fewer than $k+1$ functions, or more than $k+1$ functions? In the first case, results for the full range of possible exponents are easy to obtain, and represent a step in the proof of our theorem (see Lemma 7, for the case of $k$ functions; the case of fewer functions follows in a similar way). For the second case, some partial results are known (see $[\mathrm{G}]$ ), but under the crucial restriction that $r \geq 1$. The interesting question if there are results when $r<1$ in this case remains open.

(2) We may ask what happens when we replace the fractional integral kernel $K\left(x_{1}, \ldots, x_{k}\right)=\left|\left(x_{1}, \ldots, x_{k}\right)\right|^{-k n+\alpha}$ by a Calderón-Zygmund kernel $K$ on $\mathbb{R}^{n k}$. The issue is then the question of extending the Lacey-Thiele results to this $(k+1)$-linear context. Incidentally, there is an analogous problem in the $k$-linear case. For the range $r>1$ the desired estimates go back to the work of [C-M]. We show (in Theorem 8) how some of their ideas can be adapted to obtain the corresponding results for the full range of exponents.

Recently, after this work was completed, we learned of independent but related work of Grafakos and Kalton in this area. Their work will appear in [G-K]. 
We are grateful to L. Grafakos for pointing out a missing hypothesis in our original statement of Theorem 3 .

We now turn to the precise statements, and proofs of our results.

\section{Main Results}

Fix $k \in \mathbb{N}$. For each $1 \leq i \leq k+1,1 \leq j \leq k+1$, we are given an $n \times n$ matrix $A_{i j}$. We then define linear mappings $\ell_{j}: \mathbb{R}^{n(k+1)} \rightarrow \mathbb{R}^{n}, 1 \leq j \leq k+1$, by

$$
\ell_{j}\left(x_{1}, x_{2}, \ldots, x_{k}, x\right)=A_{1 j} x_{1}+A_{2 j} x_{2}+\cdots A_{k j} x_{k}+A_{k+1} x .
$$

We let $A=\left(A_{i j}\right)_{\substack{i=1, \ldots, k+1 \\ j=1, \ldots, k+1}}$ be the corresponding $(k+1) n \times(k+1) n$ matrix. For $0<\alpha<k n$, we define

$$
\begin{aligned}
& I_{\alpha, A}\left(f_{1}, \ldots, f_{k+1}\right)(x)= \\
& \quad \int_{\mathbb{R}^{n}} f_{1}\left(\ell_{1}\left(x_{1}, \ldots, x_{k}, x\right)\right) \cdots f_{k+1}\left(\ell_{k+1}\left(x_{1}, \ldots, x_{k}, x\right)\right) \frac{d x_{1} \cdots d x_{k}}{\left|\left(x_{1}, \ldots, x_{k}\right)\right|^{n k-\alpha}} .
\end{aligned}
$$

The functions $f_{i}, 1 \leq i \leq k+1$, will be in $L^{p_{i}}\left(\mathbb{R}^{n}\right)$, with $1 \leq p_{i} \leq \infty$, and with

$$
\frac{1}{p_{1}}+\frac{1}{p_{2}}+\cdots+\frac{1}{p_{k+1}}>\frac{\alpha}{n}
$$

Let

$$
\frac{1}{q}=\frac{1}{p_{1}}+\frac{1}{p_{2}}+\cdots \frac{1}{p_{k+1}}-\frac{\alpha}{n},
$$

and note that we are allowing for the possibility that $q<1$.

We will make the following assumptions about $A$.

(H1) For each $1 \leq j \leq k+1, A_{k+1}$ is an invertible $n \times n$ matrix.

(H2) $A$ is an invertible $(k+1) n \times(k+1) n$ matrix.

(H3) For each $j_{0}, 1 \leq j_{0} \leq k+1$, consider the $k n \times k n$ matrix $A_{j_{0}}=\left(A_{j_{0}}\right)_{l m}$, where

$$
\left(A_{j_{0}}\right)_{\ell m}= \begin{cases}A_{\ell, m} & 1 \leq \ell \leq k, 1 \leq m \leq k, m<j_{0} \\ A_{\ell, m+1} & 1 \leq \ell \leq k, 1 \leq m \leq k, j_{0} \leq m\end{cases}
$$

We assume that, for each $1 \leq j_{0} \leq k+1, A_{j_{0}}$ is an invertible $k n \times k n$ matrix. Our main result is:

Theorem 1. Assume that (H1), (H2), (H3) hold. Then,

(a) If $1<p_{i}, i=1, \ldots, k+1$,

$$
\left\|I_{\alpha, A}\left(f_{1}, \ldots, f_{k+1}\right)\right\|_{L^{q}\left(\mathbb{R}^{n}\right)} \leq C \prod_{i=1}^{k+1}\left\|f_{i}\right\|_{L^{p_{i}\left(\mathbb{R}^{n}\right)}} .
$$


(b) If at least one $p_{i}$ equals one, then

$$
\left\|I_{\alpha, A}\left(f_{1}, \ldots, f_{k+1}\right)\right\|_{L^{q, \infty}\left(\mathbb{R}^{n}\right)} \leq C \prod_{i=1}^{k+1}\left\|f_{i}\right\|_{L^{p_{i}\left(\mathbb{R}^{n}\right)}} .
$$

(Here $L^{q, \infty}$ denotes the "weak type" $L^{q}$ space; see $[S-W]$ for the definition of the Lorentz spaces $L^{q, \infty}$.)

To illustrate this result, consider the case $k=1$, and suppose that $A_{11}=I$, $A_{21}=I, A_{12}=-I, A_{22}=I$. It is then easy to see that (H1), (H2) and (H3) hold. The resulting bilinear operator is

$$
B_{\alpha}(f, g)(x)=\int f(x+t) g(x-t) \frac{d t}{|t|^{n-\alpha}}, \quad 0<\alpha<n,
$$

and a special case of Theorem 1 is:

Theorem 2. Assume that $0<\alpha<n, \frac{1}{p_{1}}+\frac{1}{p_{2}}>\frac{\alpha}{n}, \frac{1}{q}=\frac{1}{p_{1}}+\frac{1}{p_{2}}-\frac{\alpha}{n}$, and that $f \in L^{p_{1}}, g \in L^{p_{2}}, 1 \leq p_{i} \leq \infty$. Then,

(a) If $1<p_{i}, i=1,2$,

$$
\left\|B_{\alpha}(f, g)\right\|_{L^{q}} \leq C\|f\|_{L^{p_{1}}}\|g\|_{L^{p_{2}}} .
$$

(b) If $1 \leq p_{i}, i=1,2$, and either $p_{1}$ or $p_{2}$ is one,

$$
\left\|B_{\alpha}(f, g)\right\|_{L^{q, \infty}} \leq C\|f\|_{L^{p_{1}}}\|g\|_{L^{p_{2}}} .
$$

In order to illustrate the ideas in the proof of Theorem 1, we will sketch first the proof of Theorem 2. We will find very useful in the sequel the following multilinear interpolation theorem for Lorentz spaces.

Theorem 3 (S. Janson $[\mathrm{J}]$ ). Suppose that an $\ell$-linear operator $T: L^{p_{1 j, 1}} \times$ $\cdots \times L^{p_{\ell j, 1}} \rightarrow L^{q_{j}, \infty}$, where $0<p_{i_{j}} \leq \infty, 0<q_{j} \leq \infty$, for $\ell+1$ points $\left(\frac{1}{p_{1 j}}, \ldots, \frac{1}{p_{\ell j}}\right), 1 \leq j \leq \ell+1$ in $\mathbb{R}^{\ell}$, that do not lie on the same hyperplane. Suppose further that there are real numbers $\alpha_{0}, \alpha_{1}, \ldots, \alpha_{\ell}$ with $\alpha_{i}>0$ for $i=$ $1, \ldots, \ell$, so that $1 / q_{j}=\alpha_{0}+\sum_{i=1}^{\ell} \alpha_{i} / p_{i j}$, for $j=1, \ldots, \ell+1$. Then

$$
T: L^{p_{1}, s_{1}} \times \cdots \times L^{p_{\ell}, s_{\ell}} \rightarrow L^{q, s},
$$

where $1 \leq s_{j} \leq \infty, \frac{1}{s}=\frac{1}{s_{1}}+\cdots \frac{1}{s_{\ell}}$, and $\left(\frac{1}{p_{1}}, \frac{1}{p_{2}}, \ldots, \frac{1}{p_{\ell}}, \frac{1}{q}\right)$ lies in the open convex hull of $\left(\frac{1}{p_{1 j}}, \frac{1}{p_{2 j}}, \ldots, \frac{1}{p_{\ell j}}, \frac{1}{q_{j}}\right)$.

Remark 4. If $p_{j} \geq 1$, and $\frac{1}{p_{1}}+\frac{1}{p_{2}}+\ldots \frac{1}{p_{\ell}} \geq \frac{1}{q}$, since we can take $s_{j}=p_{j}$, and $\frac{1}{s} \geq \frac{1}{q}$, so that $L^{q, s} \subset L^{q}$, we obtain that $T: L^{p_{1}} \times \cdots \times L^{p_{\ell}} \rightarrow L^{q}$. 
Sketch of the proof of Theorem 2. We first establish (b) when $p_{1}=p_{2}=1$. We need a preliminary result. Let

$$
\begin{gathered}
B(f, g)(x)=\int_{|t| \leq 1} f(x+t) g(x-t) d t, \\
B_{k}(f, g)(x)=\int_{|t| \leq 2^{-k}} f(x+t) g(x-t) d t .
\end{gathered}
$$

Lemma 5. The following statements hold:

(ii) $\|B(f, g)\|_{L^{1}} \leq C\|f\|_{L^{1}}\|g\|_{L^{1}}$.

(iii) $\left\|B_{k}(f, g)\right\|_{L^{\frac{1}{2}}} \leq C 2^{-n k}\|f\|_{L^{1}}\|g\|_{L^{1}}$.

(iv) $\left\|B_{k}(f, g)\right\|_{L^{1}} \leq C\|f\|_{L^{1}}\|g\|_{L^{1}}$.

Proof. We assume, without loss of generality, that $f \geq 0, g \geq 0$.

$$
\int_{\mathbb{R}^{n}} B(f, g)(x) d x=\int_{|t| \leq 1} \int f(x+t) g(x-t) d x d t .
$$

Using the change of variables $y=x+t, z=x-t$, (ii) follows. (iv) follows by the same argument. We next establish $(i)$. For $\vec{a} \in \mathbb{Z}^{n}$, we let $Q_{\vec{a}}$ be the unit size cube in $\mathbb{R}^{n}$, whose bottom left coordinate is $\vec{a}$, and we let $Q_{\vec{a}}^{*}$ be $Q_{\vec{a}}$, expanded five times. We let $F=B(f, g), f_{\vec{a}}=\int_{Q_{\vec{a}}} f, g_{\vec{a}}=\int_{Q_{\vec{a}}} g$. Then

$$
F_{\vec{a}}=\int_{Q_{\vec{a}}} F=\int_{Q_{\vec{a}}} \int_{|t| \leq 1} f(x+t) g(x-t) d t d x \leq C \int_{Q_{\vec{a}}^{*}} f \cdot \int_{Q_{\vec{a}}^{*}} g,
$$

where we have used the same change of variables as before. Thus,

$$
F_{\vec{a}} \leq C f_{\vec{a}}^{*} g_{\vec{a}}^{*}, \quad \text { where } f_{\vec{a}}^{*}=\int_{Q_{\vec{a}}^{*}} f, g_{\vec{a}}^{*}=\int_{Q_{\vec{a}}^{*}} g
$$

Since $\int_{Q_{\vec{a}}} F^{\frac{1}{2}} \leq\left(F_{\vec{a}}\right)^{\frac{1}{2}} \leq C\left(f_{\vec{a}}^{*}\right)^{\frac{1}{2}}\left(g_{\vec{a}}^{*}\right)^{\frac{1}{2}}$, we obtain that

$$
\int F^{\frac{1}{2}} \leq C\left(\sum_{\vec{a}} f_{\vec{a}}^{*}\right)^{\frac{1}{2}}\left(\sum_{\vec{a}} g_{\vec{a}}^{*}\right)^{\frac{1}{2}} .
$$

Finally since the $\left\{Q_{\vec{a}}^{*}\right\}$ have finite overlap, $\sum_{\vec{a}} f_{\vec{a}}^{*} \leq C\|f\|_{L^{1}}$, and similarly for $g$, and $(i)$ follows. (iii) follows from $(i)$ by scaling, and Lemma 5 is established.

Let $p_{1}=1, p_{2}=1, \frac{1}{q}=2-\frac{\alpha}{n}$. Let

$$
F_{k}(x)=\int_{|t| \simeq 2^{-k}} f(x+t) g(x-t) \frac{d t}{|t|^{n}},
$$


so that

$$
B_{\alpha}(f, g)(x) \leq \sum_{k} 2^{-k \alpha} F_{k}(x)=H(x) .
$$

Note that Lemma $5(i i i)$ and $(i v)$ give

$$
\left\|F_{k}\right\|_{L^{\frac{1}{2}}} \leq C\|f\|_{L^{1}}\|g\|_{L^{1}}, \quad\left\|F_{k}\right\|_{L^{1}} \leq C 2^{k n}\|f\|_{L^{1}}\|g\|_{L^{1}}
$$

respectively. Write $H(x)=F_{1}+F_{2}$, where $F_{1}=\sum_{k \leq k_{0}} 2^{-k \alpha} F_{k}(x), F_{2}=$ $\sum_{k>k_{0}} 2^{-k \alpha} F_{k}(x)$, so that

$$
\begin{gathered}
\left\|F_{1}\right\|_{L^{1}} \leq C 2^{k_{0}(n-\alpha)}\|f\|_{L^{1}}\|g\|_{L^{1}}, \text { and } \\
\left\|F_{2}\right\|_{L^{\frac{1}{2}}}^{2} \leq \sum_{k>k_{0}} 2^{-k \alpha / 2}\left\|F_{k}(x)\right\|_{L^{\frac{1}{2}}}^{2} \leq C 2^{-k_{0} \alpha / 2}\|f\|_{L^{1}}^{2}\|g\|_{L^{1}}^{2} .
\end{gathered}
$$

Then,

$$
\begin{aligned}
\left|\left\{B_{\alpha}(f, g)>\lambda\right\}\right| & \leq\left|\left\{F_{1} \geq \frac{C \lambda}{2}\right\}\right|+\left|\left\{F_{2} \geq \frac{C \lambda}{2}\right\}\right| \\
& \leq \frac{C}{\lambda} 2^{k_{0}(n-\alpha)}\|f\|_{L^{1}}\|g\|_{L^{1}}+\frac{C}{\lambda^{\frac{1}{2}}} 2^{\frac{-k_{0} \alpha}{2}}\|f\|_{L^{1}}^{2}\|g\|_{L^{1}}^{2} .
\end{aligned}
$$

Note that we can assume that $\|g\|_{L^{1}}=\|f\|_{L^{1}}=1$ and choose $k_{0}$ so that $2^{k_{0}(n-\alpha)} / \lambda \sim 2^{-k_{0} \alpha / 2} / \lambda^{\frac{1}{2}}$. This gives the estimate $C / \lambda^{q}, \frac{1}{q}=2-\frac{\alpha}{n}$ as desired.

To finish the proof of (b), note that, if $g \in L^{\infty}$, we have

$$
B_{\alpha}(f, g)(x) \leq\|g\|_{L^{\infty}} \int f(x+t) \frac{d t}{|t|^{n-\alpha}},
$$

and so (b) follows in this case by ordinary fractional integration. If $g \in L^{p_{2}}$, $1<p_{2}<\infty, f \in L^{1}$, we obtain the desired result (b) by fixing $f$ and using Theorem 3 in $g$, with $\ell=1$. In this case, note that $\frac{1}{q}>\frac{1}{p_{2}}$, so we can only conclude that $B_{\alpha}(f, g) \in L^{q, \infty}$ (in fact, $\left.L^{q, p_{2}}\right)$. To obtain (a), we use Remark 4, this time with $\ell=2$. In fact, consider the open convex set in $\mathbb{R}^{2}$,

$$
C=\left\{\left(z_{1}, z_{2}\right): z_{1}+z_{2}>\frac{\alpha}{n}, 0<z_{1}<1,0<z_{2}<1\right\} .
$$

Then, since $\frac{\alpha}{n}<1$, the closure of $C$ meets each side of the square $0 \leq z_{1} \leq 1$, $0 \leq z_{2} \leq 1$, and for each pair of points on different sides of the square intersected with the closure of $C$, we can find a third point, on a third side of the square intersected with the closure of $C$, and thus, not collinear with the first two points. Moreover, the interior of the convex hull of all such pairs of points is exactly $C$. Therefore, in view of Remark 4, which applies to our situation, the preceding remarks, and symmetry, it suffices to show the "weak type" inequality for $p_{1}=1, \infty$ and $1<p_{2}<\infty$. The case $p_{1}=1$ is covered by (b), while the case $p_{1}=\infty$ holds because

$$
B_{\alpha}(f, g)(x) \leq\|f\|_{L^{\infty}} \int g(x-t) \frac{d t}{|t|^{n-\alpha}},
$$


and so (a) follows in this case by ordinary fractional integration. Theorem 2 is now completely proved.

Remark 6. When $g(x)=\delta_{0}(x), B_{\alpha}(f, g)(x)=f(2 x) /|x|^{n-\alpha}$, which shows that in (b) the strong type inequality fails.

We now turn to the proof of Theorem 1. The general scheme of the proof is identical to the one we just gave for Theorem 2. We will need a further multilinear extension of ordinary fractional integration.

Lemma 7. Let $k \in \mathbb{N}$,

$$
\frac{1}{s}=\frac{1}{r_{1}}+\frac{1}{r_{2}}+\cdots+\frac{1}{r_{k}}-\frac{\alpha}{n}>0,
$$

with $0<\alpha<k n, 1 \leq r_{i} \leq \infty$, and define

$$
I_{\alpha, k}\left(f_{1}, \ldots, f_{k}\right)(x)=\int \frac{f_{1}\left(x-y_{1}\right) f_{2}\left(x-y_{2}\right) \cdots f_{k}\left(x-y_{k}\right)}{\left|\left(y_{1}, \ldots, y_{k}\right)\right|^{k n-\alpha}} d y_{1} \cdots d y_{k} .
$$

Then,

(a) If each $r_{i}>1$,

$$
\left\|I_{\alpha, k}\left(f_{1}, \ldots, f_{k}\right)\right\|_{L^{s}} \leq C \prod_{i=1}^{k}\left\|f_{i}\right\|_{L^{r_{i}}} .
$$

(b) If $r_{i}=1$ for some $i$,

$$
\left\|I_{\alpha, k}\left(f_{1}, \ldots, f_{k}\right)\right\|_{L^{s, \infty}} \leq C \prod_{i=1}^{k}\left\|f_{i}\right\|_{L^{r_{i}}} .
$$

We will take Lemma 7 temporarily for granted, and use it to give the proof of Theorem 1. We will then return to the proof of Lemma 7.

Proof of Theorem 1. We first establish (b) when $p_{1}=p_{2}=\cdots=p_{k+1}=1$. The general scheme is identical to the one used in the proof of Theorem 2 . We let

$$
\begin{aligned}
& M_{A}\left(f_{1}, f_{2}, \ldots, f_{k+1}\right)(x)= \\
& \int_{\left|\left(x_{1}, \ldots, x_{k}\right)\right| \leq 1} f_{1}\left(\ell_{1}\left(x_{1}, \ldots, x_{k}, x\right)\right) \cdots f_{k+1}\left(\ell_{k+1}\left(x_{1}, \ldots, x_{k}, x\right)\right) d x_{1} \cdots d x_{k},
\end{aligned}
$$

and,

$$
\begin{aligned}
& M_{A, s}\left(f_{1}, f_{2}, \ldots, f_{k+1}\right)(x)= \\
& \int_{\left|\left(x_{1}, \ldots, x_{k}\right)\right| \leq 2^{-s}} f_{1}\left(\ell_{1}\left(x_{1}, \ldots, x_{k}, x\right)\right) \cdots f_{k+1}\left(\ell_{k+1}\left(x_{1}, \ldots, x_{k}, x\right)\right) d x_{1} \cdots d x_{k},
\end{aligned}
$$

and, we have the analogue of Lemma 5: 
$\begin{aligned} \text { (i) }\left\|M_{A}\left(f_{1}, \ldots, f_{k+1}\right)\right\|_{L^{\frac{1}{k+1}}} & \leq C \prod_{r=1}^{k+1}\left\|f_{r}\right\|_{L^{1}}, \\ \text { (ii) }\left\|M_{A}\left(f_{1}, \ldots, f_{k+1}\right)\right\|_{L^{1}} & \leq C \prod_{r=1}^{k+1}\left\|f_{r}\right\|_{L^{1}}, \\ \text { (iii) }\left\|M_{A, s}\left(f_{1}, \ldots, f_{k+1}\right)\right\|_{L^{\frac{1}{k+1}}} & \leq C 2^{-n s k} \prod_{r=1}^{k+1}\left\|f_{r}\right\|_{L^{1}}, \\ \text { (iv) }\left\|M_{A, s}\left(f_{1}, \ldots, f_{k+1}\right)\right\|_{L^{1}} & \leq C \prod_{r=1}^{k+1}\left\|f_{r}\right\|_{L^{1}} .\end{aligned}$

(ii) and (iv) follow by considering the change of variables $y=L\left(x_{1}, \ldots, x_{k}, x\right)$, $y \in \mathbb{R}^{(k+1) n}$, where

$$
y_{1}=\ell_{1}\left(x_{1}, \ldots, x_{k}, x\right), \ldots, y_{k+1}=\ell_{k+1}\left(x_{1}, \ldots, x_{k}, x\right) .
$$

Hypothesis (H2) guarantees that $L$ is an isomorphism, and thus (ii) and (iv) follow as in Lemma 5. (iii) follows from (i) by rescaling. Finally, if $Q_{\vec{a}}$ is as in the proof of Lemma $5, x_{\vec{a}}$ is the center of $Q_{\vec{a}}$, and

$$
y_{\vec{a}}=L\left(0,0, \ldots, 0, x_{\vec{a}}\right)=\left(A_{k+11} x_{\vec{a}}, A_{k+12} x_{\vec{a}}, \ldots, A_{k+1 k+1} x_{\vec{a}}\right),
$$

then, if for each $j=1, \ldots, k+1$, we let $Q_{\vec{a}, j}^{*}=A_{k+1} x_{\vec{a}}+Q^{*}$, where $Q^{*}$ is a cube centered at the origin, of side length $M$, we can choose $M$, depending only on $n, k, A$, so that

$$
L\left(\left\{\left|\left(x_{1}, \ldots, x_{k}\right)\right| \leq 1\right\} \times Q_{\vec{a}}\right) \subset Q_{\vec{a}, 1}^{*} \times \cdots \times Q_{\vec{a}, k+1}^{*} .
$$

Moreover, since (H1) holds, the cubes $Q_{\vec{a}, j}^{*}$ have finite overlap, depending only on $n, k, A$, and this gives (i) just as in the proof of Lemma 5 . Next, with

$$
\begin{aligned}
& F_{s}(x)=\int_{\left|\left(x_{1}, \ldots, x_{k}\right)\right| \simeq 2^{-s}} f_{1}\left(\ell_{1}\left(x_{1}, \ldots, x_{k}, x\right)\right) \cdots \\
& \cdots f_{k+1}\left(\ell_{k+1}\left(x_{1}, \ldots, x_{k}, x\right)\right) \frac{d x_{1} \cdots d x_{k}}{\left|\left(x_{1}, \ldots, x_{k}\right)\right|^{k n}},
\end{aligned}
$$

we have $I_{\alpha, A}\left(f_{1}, \ldots, f_{k+1}\right)(x) \leq \sum_{s} 2^{-s \alpha} F_{s}(x)=H(x)$, and

$$
\left\|F_{s}\right\|_{L^{\frac{1}{k+1}}} \leq C \prod_{r=1}^{k+1}\left\|f_{r}\right\|_{L^{1}},\left\|F_{s}\right\|_{L^{1}} \leq C 2^{s k n} \prod_{r=1}^{k+1}\left\|f_{r}\right\|_{L^{1}} .
$$

We will then write $H(x)=F_{1}+F_{2}$,

$$
F_{1}=\sum_{s \leq s_{0}} 2^{-s \alpha} F_{s}(x), F_{2}=\sum_{s>s_{0}} 2^{-s \alpha} F_{s}(x),
$$

and choose $s_{0}$ so that

$$
\frac{2^{s_{0}(k n-\alpha)}}{\lambda}=\frac{2^{-s_{0} \alpha / k+1}}{\lambda^{1 / k+1}},
$$

to obtain $\left|\left\{I_{\alpha, A}>\lambda\right\}\right| \leq \frac{C}{\lambda^{q}}, \frac{1}{q}=(k+1)-\frac{\alpha}{n}$, as desired. To finish up the proof of (b) in Theorem 1, we proceed inductively. We will assume that the statement in (b) is proved when $j+1$ of the $p_{i}$ 's are equal to one, and then prove (b) when $j$ of the $p_{i}$ 's equal one. Since we have just shown that (b) holds when all the $p_{i}$ are 1 , this suffices. Thus assume (by symmetry), $p_{1}=1, p_{2}=1, \ldots, p_{j}=1,1<$ 
$p_{j+1} \leq \infty, \ldots, 1<p_{k+1} \leq \infty$, with $j+\frac{1}{p_{j+1}}+\cdots+\frac{1}{p_{k+1}}>\frac{\alpha}{n}$. We can assume $1 \leq j \leq k$. Set $\frac{1}{q}=j+\frac{1}{p_{j+1}}+\cdots+\frac{1}{p_{k+1}}-\frac{\alpha}{n}$, and fix $f_{1}, \ldots, f_{j} \in L^{1}$. With $\ell=k+1-j$, we consider the $\ell$-linear operator

$$
T\left(g_{1}, g_{2}, \ldots, g_{\ell}\right)(x)=I_{\alpha, A}\left(f_{1}, \ldots, f_{j}, g_{1}, \ldots, g_{\ell}\right)(x) .
$$

We will use Theorem 3 to show that

$$
T: L^{p_{j+1}} \times L^{p_{j+2}} \times \cdots \times L^{p_{k+1}} \rightarrow L^{q, \infty} .
$$

Consider the open convex set in $\mathbb{R}^{\ell}$,

$$
C=\left\{\left(z_{1}, \ldots, z_{\ell}\right): j+z_{1}+z_{2}+\cdots+z_{\ell}>\frac{\alpha}{n}, 0<z_{i}<1, i=1, \ldots, \ell\right\} .
$$

We will first assume that $\ell>1$. First, note that $C$ is the open convex hull of those points in the faces of $Q_{0}=\left\{\left(z_{1} \ldots, z_{\ell}\right): 0 \leq z_{i} \leq 1,1 \leq i \leq \ell\right\}$ which are also in the half-plane $H=\left\{\left(z_{1} \ldots, z_{\ell}\right): j+z_{1}+z_{2}+\cdots+z_{\ell}>\frac{\alpha}{n}\right\}$. Moreover, if $\vec{z} \in C$, and $\vec{z}_{1}, \vec{z}_{2}$ are each in the intersection of a face of $Q_{0}$ with $H$, and $\vec{z}$ belongs to the line segment between $\vec{z}_{1}$ and $\vec{z}_{2}$, then $\vec{z}_{1}$ and $\vec{z}_{2}$ must belong to different faces of $Q_{0}$, and hence must be linearly independent. Also note that $H$ meets the interior of each of the $2 \ell$ faces of $Q_{0}$ in a non-empty open subset. (Here and in the sequel, when referring to the interior of a face, or an open subset of a face, we mean this as subsets of the $\mathbb{R}^{\ell-1}$ dimensional hyperplane which contains the face.) Next, observe that, given any pair of points $\vec{z}_{1}=\left(z_{1}^{1}, \ldots, z_{\ell}^{1}\right), \vec{z}_{2}=\left(z_{1}^{2}, \ldots, z_{\ell}^{2}\right)$, each in the intersection of a different face of $Q_{0}$ with $H$ (and hence linearly independent) we can find $\ell-2$ points $\vec{z}_{3}, \vec{z}_{4}, \ldots, \vec{z}_{\ell}$ so that each point is in the intersection of a different face of $Q_{0}$ with $H$, and so that $\left\{\vec{z}_{1}, \vec{z}_{2}, \ldots, \vec{z}_{\ell}\right\}$ is linearly independent.

(To see this, recall that $\vec{z}_{1}$ and $\vec{z}_{2}$ are linearly independent. If $L_{2}$ is the 2dimensional subspace that they span, and $F_{3}$ is any face of $Q_{0}$ different from the faces $F_{1}$ and $F_{2}$ to which $\vec{z}_{1}$ and $\vec{z}_{2}$ belong, then $L_{2}$ meets $F_{3}$ along an affine space $A_{2}$. Note that $L_{2}$ cannot be contained in the $(\ell-1)$ dimensional hyperplane containing $F_{3}$ (since $\vec{z}_{1}, \vec{z}_{2} \notin F_{3}$ ), and so, $\operatorname{dim} A_{2} \leq 1$. Since $\ell-2>0$ (otherwise there is nothing to do), $\ell-1>1$, and hence can find a point $\vec{z}_{3}$ in the interior of $F_{3}$ intersected with $H$, which is not in $A_{2}$. Thus, $\left\{\vec{z}_{1}, \vec{z}_{2}, \vec{z}_{3}\right\}$ are linearly independent. We continue in this fashion, until we have $\ell$ points. This is possible because if we have $\left\{\vec{z}_{1}, \vec{z}_{2}, \ldots, \vec{z}_{r}\right\}$ linearly independent, each in a different face, and if we let $L_{r}$ be their linear span, and $F_{r+1}$ is a further face, and

$$
A_{r}=L_{r} \cap\left(\ell-1 \text { dimensional hyperplane containing } F_{r+1}\right),
$$

then $A_{r} \stackrel{\subsetneq}{\neq} L_{r}, \operatorname{dim}\left(L_{r}\right)=r$, so if $r \leq \ell-1$, then $\operatorname{dim} A_{r} \leq r-1 \leq \ell-2$, and thus we can find $\vec{z}_{r+1}$ in the interior of $F_{r+1}$ intersected with $H$, and not in $A_{r}$.)

By linear independence, there exists a unique hyperplane $L$ in $\mathbb{R}^{\ell}$, which contains $\vec{z}_{1}, \vec{z}_{2}, \ldots, \vec{z}_{\ell}$. Let $F_{\ell+1}$ be a face of $C$ different from the ones containing $\vec{z}_{1}, \ldots, \vec{z}_{\ell}$. Then $L$ cannot be contained in the hyperplane containing $F_{\ell+1}$, and 
so the dimension of the intersection of $L$ with that hyperplane does not exceed $\ell-2$. Hence, we can find a further point $\vec{z}_{\ell+1}$ in the interior of $F_{\ell+1}$ intersected with $H$, which is not $L$. By the uniqueness of $L,\left\{\vec{z}_{1}, \vec{z}_{2}, \ldots, \vec{z}_{\ell}, \vec{z}_{\ell+1}\right\}$ are not in the same hyperplane.

Because of the above considerations, Theorem 3, and symmetry, we need only show our weak type estimate when $\left(\frac{1}{p_{j+1}}, \ldots, \frac{1}{p_{k+1}}\right)=\left(z_{1}, \ldots, z_{\ell}\right)$ is such that $z_{1}$ is either 0 or $1,0 \leq z_{i}<1, i=1,2, \ldots, \ell$.

If $z_{1}=0, g_{1} \in L^{\infty}$, and

$$
\begin{aligned}
& T\left(g_{1}, \ldots, g_{\ell}\right)(x) \leq \\
& \left\|g_{1}\right\|_{L^{\infty}} \int f_{1}\left(\ell_{1}\left(x_{1}, \ldots, x_{k}, x\right)\right) \cdots f_{j}\left(\ell_{j}\left(x_{1}, \ldots, x_{k}, x\right)\right) \\
& g_{2}\left(\ell_{j+2}\left(x_{1}, \ldots, x_{k}, x\right)\right) \cdots g_{\ell}\left(\ell_{k+1}\left(x_{1}, \ldots, x_{k}, x\right)\right) \frac{d x_{1} \cdots d x_{k}}{\left|\left(x_{1}, \ldots, x_{k}\right)\right|^{n k-\alpha}} .
\end{aligned}
$$

At this point, hypotheses (H1) and (H3) easily reduce matters to Lemma 7. (See Remark 10 below.)

If $z_{1}=1, g_{1} \in L^{1}$, and the required bound now follows from the fact that $j+1$ of the $p_{i}$ 's are equal to one.

If $\ell=1$, we only require two points to apply Theorem 3, and the argument is much simpler, and thus (b) is established.

We now turn to the proof of (a) in Theorem 1. As in the proof of Theorem 2 , we will use Remark 4 . The argument will be similar to the one used above to give the proof of (b). Consider the open convex set in $\mathbb{R}^{k+1}$,

$$
C=\left\{\left(z_{1}, \ldots, z_{n}\right): z_{1}+z_{2}+\cdots+z_{k+1}>\alpha / n, 0<z_{i}<1, i=1, \ldots, k+1\right\},
$$

where, as always, $0<\alpha<k n$. The half-plane $H=\left\{z_{1}+\cdots+z_{k+1}>\alpha / n\right\}$ meets the interior of each face of the cube $Q_{0}=\left\{0<z_{i}<1, i=1, \ldots, k+1\right\}$ in an open set, and thus, arguing as in the proof of (b), but this time using Remark 4 (since $\frac{1}{q}<\frac{1}{p_{1}}+\cdots+\frac{1}{p_{k+1}}$ ), we are reduced to showing the "weak type" estimate, when $p_{1}=1$ or $\infty, 1<p_{j} \leq \infty, j=2, \ldots, k+1$,

$$
\frac{1}{p_{1}}+\frac{1}{p_{2}}+\cdots+\frac{1}{p_{k+1}}-\frac{\alpha}{n}>0 \text {. }
$$

When $p_{1}=\infty$, hypotheses (H1) and (H3) quickly reduce matters to Lemma 7 (see Remark 10 below), and when $p_{1}=1$ the desired result follows from (b).

Proof of Lemma \%. Since $\alpha>0$, some $r_{i}<\infty$. If, say, $r_{\ell+1}=\cdots=r_{k}=\infty$, $1 \leq \ell<k$, because $\frac{\alpha}{n}<\frac{1}{r_{1}}+\cdots+\frac{1}{r_{\ell}} \leq \ell$, so that $k n-\alpha>(k-\ell) n$, integration in $y_{\ell+1}, \ldots, y_{k}$ reduces matters to the case when all $r_{i}$ are finite (and $k=\ell$ ). Thus, we can assume that all $r_{i}<\infty$. Now, observe that if $0<c_{i}, i=1, \ldots, k$, and $0<\alpha<\sum_{i=1}^{k} c_{i}$, we can find $0<\alpha_{i}<c_{i}$ such that $\alpha=\sum_{i=1}^{k} \alpha_{i}$. Apply 
this observation to $c_{i}=n / r_{i}$, and let $\frac{1}{s_{i}}=\frac{1}{r_{i}}-\frac{\alpha_{i}}{n}$. Since $\sum_{i=1}^{k} \frac{1}{s_{i}}=\frac{1}{s}, 0<$ $\frac{\alpha_{i}}{n}<\frac{1}{r_{i}} \leq 1,1<s_{i}<\infty$, and

$$
\left|y_{1}\right|^{n-\alpha_{1}}\left|y_{2}\right|^{n-\alpha_{2}} \cdots\left|y_{k}\right|^{n-\alpha_{k}} \leq\left|\left(y_{1}, \ldots, y_{k}\right)\right|^{n k-\alpha}\left(\alpha=\sum_{i=1}^{k} \alpha_{i}\right),
$$

it follows that $I_{\alpha, k}\left(f_{1}, \ldots, f_{k}\right)(x) \leq \prod_{i=1}^{k} I_{\alpha_{i}}\left(f_{i}\right)(x)$, where $I_{\alpha_{i}}$ is the standard fractional integral operator. The lemma now follows by Hölder's inequality (or its Lorentz space version when some $r_{i}=1$ ).

\section{A Singular Integral Version}

We will conclude with a "singular integral" version of Lemma 7, which allows one to extend to exponents below 1, results of Coifman-Meyer [C-M1, C-M2, C-M3, C-M4, C-M5].

Theorem 8. Let $k \in \mathbb{N}, 1 \leq r_{i}<\infty, 1 \leq i \leq k$,

$$
\frac{1}{s}=\frac{1}{r_{1}}+\cdots+\frac{1}{r_{k}}
$$

and let $K$ denote a Calderón-Zygmund kernel in $\mathbb{R}^{n k}$ (i.e. $K$ is homogeneous of degree $-n k$, it is smooth away from the origin, and has mean value 0 on the unit sphere in $\left.\mathbb{R}^{n k}\right)$. For $f_{i} \in L^{r_{i}}$, let

$$
T_{K}\left(f_{1}, \ldots, f_{k}\right)(x)=p . v \cdot \int f_{1}\left(x-y_{1}\right) \cdots f_{k}\left(x-y_{k}\right) K\left(y_{1}, \ldots, y_{k}\right) d y_{1} \cdots d y_{k} .
$$

Then:

(a) If each $r_{i}>1$, then

$$
\left\|T_{K}\left(f_{1}, \ldots, f_{k}\right)\right\|_{L^{s}} \leq C \prod_{i=1}^{k}\left\|f_{i}\right\|_{L^{r_{i}}} .
$$

(b) If $r_{i}=1$ for some $i$, then

$$
\left\|T_{K}\left(f_{1}, \ldots, f_{k}\right)\right\|_{L^{s, \infty}} \leq C \prod_{i=1}^{k}\left\|f_{i}\right\|_{L^{r_{i}}}
$$

Remark 9. When $r_{i}>1, s>1$, (a) is due to Coifman-Meyer [C-M1], [C-M2], who also proved that if $s=1$, the weak-type estimate holds. Note that in [C-M3], [C-M4] it is also allowed that $(k-1)$ of the $r_{i}$ be $\infty$. Our point here is the extension to the case $s<1$, and also adapts ideas used by them, in particular Lemma 4.8 in [C-M1]. 
Proof of Theorem 8. We will only do the $k=2$ case here. We also do, for simplicity, the case $n=1$. We first prove the case $s=\frac{1}{2}, r_{1}=1, r_{2}=1$ in (b). Thus let $f_{i} \in L^{1}$, and assume, without loss of generality, that $\left\|f_{i}\right\|_{L^{1}}=1$. Let $\lambda>0$, and we want to show that

$$
\left|\left\{x:\left|T_{K}\left(f_{1}, f_{2}\right)\right|>\lambda\right\}\right| \leq C / \lambda^{\frac{1}{2}}
$$

We next perform a Calderón-Zygmund decomposition (see $[\mathrm{S}]$ ) of $f_{i}$, at "height" $\lambda^{\frac{1}{2}}$, i.e. we write $f_{i}=b_{i}+g_{i}$, where $b_{i}=\sum_{k} b_{i, k}, \operatorname{supp} b_{i, k} \subset I_{i, k}, \int_{I_{i, k}} b_{i, k}=$ $0,\left\{I_{i, k}\right\}_{k}$ are a disjoint collection of intervals, $\left|\cup_{k} I_{i, k}\right| \leq C / \lambda^{\frac{1}{2}}, \int_{I_{i, k}}\left|b_{i, k}\right| \leq$ $C \lambda^{\frac{1}{2}}\left|I_{i, k}\right|$, and $\left\|g_{i}\right\|_{L^{\infty}} \leq C \lambda^{\frac{1}{2}}$. Note that $\int\left|b_{i}\right| \leq C$, and that, for $q>1$, $\int\left|g_{i}\right|^{q} \leq C \lambda^{\frac{q-1}{2}}$,

$$
\begin{aligned}
\left\{T_{K}\left(f_{1}, f_{2}\right)>\lambda\right\} \subset & \\
& \left\{T_{K}\left(b_{1}, b_{2}\right)>\lambda / 4\right\} \cup\left\{T_{K}\left(b_{1}, g_{2}\right)>\lambda / 4\right\} \\
& \cup\left\{T_{K}\left(g_{1}, b_{2}\right)>\lambda / 4\right\} \cup\left\{T_{K}\left(g_{1}, g_{2}\right)>\lambda / 4\right\} \\
& =E_{1} \cup E_{2} \cup E_{3} \cup E_{4} .
\end{aligned}
$$

Let $\Omega_{i}^{*}=\cup_{k} I_{i, k}{ }^{*}$, where $I_{i, k}{ }^{*}$ is the interval with the same center as $I_{i, k}$, but expanded three times.

We first estimate $E_{1}=E_{1} \backslash\left(\Omega_{1}^{*} \cup \Omega_{2}^{*}\right) \cup E_{1} \cap\left(\Omega_{1}^{*} \cup \Omega_{2}^{*}\right)$. The second of the above sets has the required estimate. Thus, assume $x \in E_{1}, x \notin\left(\Omega_{1}^{*} \cup \Omega_{2}^{*}\right)$. Then, $T_{K}\left(b_{1}, b_{2}\right)(x)=\sum_{k, j} T_{K}\left(b_{1, k}, b_{2, j}\right)(x)$. Fix $k, j$ and consider $T_{K}\left(b_{1, k}, b_{2, j}\right)(x)$. We can assume, by symmetry, that $\left|I_{1, k}\right| \leq\left|I_{2, j}\right|$. Let $u_{1, k}$ be the center of $I_{1, k}$. Then,

$$
\begin{aligned}
& T_{K}\left(b_{1, k}, b_{2, j}\right)(x)=\iint b_{1, k}\left(x-y_{1}\right) b_{2, j}\left(x-y_{2}\right) K\left(y_{1}, y_{2}\right) d y_{1} d y_{2} \\
&=\iint b_{1, k}\left(u_{1}\right) b_{2, j}\left(x-y_{2}\right) K\left(x-u_{1}, y_{2}\right) d u_{1} d y_{2} \\
&=\iint b_{1, k}\left(u_{1}\right) b_{2, j}\left(x-y_{2}\right), \\
& {\left[K\left(x-u_{1}, y_{2}\right)-K\left(x-u_{1, k}, y_{2}\right)\right] d u_{1} d y_{2} }
\end{aligned}
$$

Since for $x \notin I_{1, k}^{*},\left|K\left(x-u_{1}, y_{2}\right)-K\left(x-u_{1, k}, y_{2}\right)\right| \leq C \frac{\left|I_{1, k}\right|}{\left|\left(x-u_{1, k}, y_{2}\right)\right|^{3}}, u_{1} \in I_{1, k}$, $\left|\left(x-u_{1, k}, y_{2}\right)\right|^{3} \geq\left|x-u_{1, k}\right|^{\frac{3}{2}}\left|y_{2}\right|^{\frac{3}{2}}$ and, for $x \notin I_{1, j}^{*}, u_{2} \in I_{2, j},\left|x-u_{2}\right| \simeq\left|x-u_{2, j}\right|$, 
where $u_{2, j}$ is the center of $I_{2, j}$, we have:

$$
\begin{aligned}
\left|T_{K}\left(b_{1, k}, b_{2, j}\right)(x)\right| & \leq C \iint \frac{\left|b_{1, k}\left(u_{1}\right)\right|}{\left|x-u_{1, k}\right|^{\frac{3}{2}}}\left|I_{1, k}\right|^{\frac{1}{2}} \frac{\left|b_{2, j}\left(u_{2}\right)\right|}{\left|x-u_{2, j}\right|^{\frac{3}{2}}}\left|I_{2, j}\right|^{\frac{1}{2}} d u_{1} d u_{2}, \\
& \leq C \lambda \frac{\left|I_{1, k}\right|^{\frac{3}{2}}\left|I_{2, j}\right|^{\frac{3}{2}}}{\left|x-u_{1, k}\right|^{\frac{3}{2}}\left|x-u_{2, j}\right|^{\frac{3}{2}}}, \\
& \leq \frac{C \lambda\left|I_{1, k}\right|^{\frac{3}{2}}\left|I_{2, j}\right|^{\frac{3}{2}}}{\left(\ell\left(I_{1, k}\right)+\left|x-u_{1, k}\right|\right)^{\frac{3}{2}}\left(\ell\left(I_{2, j}\right)+\left|x-u_{2, j}\right|\right)^{\frac{3}{2}}} .
\end{aligned}
$$

Hence for $x \notin \Omega_{1}^{*} \cup \Omega_{2}^{*}$, we have:

$$
\left|T_{K}\left(b_{1}, b_{2}\right)(x)\right| \leq \sum_{j, k} \frac{\left|I_{1, k}\right|^{\frac{3}{2}}}{\left(\ell\left(I_{1, k}\right)+\left|x-u_{1, k}\right|\right)^{\frac{3}{2}}} \frac{\left|I_{2, j}\right|^{\frac{3}{2}}}{\left(\ell\left(I_{1, k}\right)+\left|x-u_{2, j}\right|\right)^{\frac{3}{2}}} \cdot C \lambda .
$$

Let

$$
\mathcal{G}_{i, \alpha}(x)=\sum_{k} \frac{\left|I_{i, k}\right|^{1+\alpha}}{\left(\ell\left(I_{i, k}\right)+\left|x-u_{i, k}\right|\right)^{1+\alpha}}, \quad \text { for } \alpha>0,
$$

be the Marcinkiewicz function, and recall (see [S]) that $\int \mathcal{G}_{i, \alpha}(x) d x \leq C_{\alpha} \mid \cup_{k}$ $I_{i, k} \mid \leq C_{\alpha} / \lambda^{\frac{1}{2}}$. We have

$$
\left|E \backslash\left(\Omega_{1}^{*} \cup \Omega_{2}^{*}\right)\right| \leq \frac{C}{\lambda^{\frac{1}{2}}} \int\left(\mathcal{G}_{1, \frac{1}{2}}(x) \cdot \mathcal{G}_{2, \frac{1}{2}}(x)\right)^{\frac{1}{2}} d x \cdot \lambda^{\frac{1}{2}} \leq C / \lambda^{\frac{1}{2}}
$$

by Cauchy-Schwarz. We estimate $E_{4}$ using the Coifman-Meyer [C-M2] result, with $r_{1}=4, r_{2}=4, s=2$, to see that

$$
\left|E_{4}\right| \leq \frac{1}{\lambda^{2}} \int\left|T_{K}\left(g_{1}, g_{2}\right)\right|^{2} \leq \frac{C}{\lambda^{2}}|| g_{1}\left\|_{L^{4}}^{2}|| g_{2}\right\|_{L^{4}}^{2} \leq \frac{C}{\lambda^{2}} \lambda^{\frac{3}{4}} \lambda^{\frac{3}{4}}=\frac{C}{\lambda^{\frac{1}{2}}} .
$$

To estimate $E_{2}$, we estimate its part disjoint from $\Omega_{1}^{*}$. If $x \notin I_{1, k}^{*}$, using the same estimate as before, we see that

$$
\begin{aligned}
\left|T_{K}\left(b_{1, k}, g_{2}\right)(x)\right| & \leq C \iint \frac{\left|b_{1, k}\left(u_{1}\right)\right|}{\left|\left(x-u_{1, k}, y_{2}\right)\right|^{3}}\left|I_{1, k}\right|\left|g_{2}\left(x-y_{2}\right)\right| d u_{1} d y_{2}, \\
& \leq C \lambda^{\frac{1}{2}} \iint \frac{\left|b_{1, k}\left(u_{1}\right)\right|\left|I_{1, k}\right|}{\left|\left(x-u_{1, k}, y_{2}\right)\right|^{3}} d u_{1} d y_{2} .
\end{aligned}
$$

Performing the $y_{2}$ integration we obtain

$$
\leq \frac{C \lambda^{\frac{1}{2}}\left|I_{1, k}\right|}{\left|\left(x-u_{1, k}\right)\right|^{2}} \int\left|b_{1, k}\left(u_{1}\right)\right| d u_{1} \leq \frac{C \lambda\left|I_{1, k}\right|^{2}}{\left(\ell\left(I_{1, k}\right)+\left|x-u_{1, k}\right|\right)^{2}} .
$$

Thus $\left|T_{K}\left(b_{1}, g_{2}\right)(x)\right| \leq C \lambda \mathcal{G}_{1,1}(x)$, and so $\left|E_{2} \backslash \Omega_{1}^{*}\right| \leq C \int \mathcal{G}_{1,1}(x) \leq C / \lambda^{\frac{1}{2}}$, as desired. Since the esimate for $E_{3}$ is similar, this concludes this case. To finish the proof of (b), fix $f_{1} \in L^{1},\left\|f_{1}\right\|_{L^{1}}=1$, and first assume that $f_{2} \in L^{\infty}$, 
$\left\|f_{2}\right\|_{L^{\infty}}=1$. Given $\lambda>0$, perform the Calderón-Zygmund decomposition of $f_{1}$, at height $\lambda$, so that $f_{1}=b_{1}+g_{1}$. By the argument above, for $x \notin \Omega_{1}^{*}$,

$$
\left|T_{K}\left(b_{1}, f_{2}\right)(x)\right| \leq C|| f_{2} \|_{L^{\infty}} \lambda \mathcal{G}_{1,1}(x),
$$

and $\int \mathcal{G}_{1,1}(x) \leq C / \lambda$. Also,

$$
\left|\left\{T_{K}\left(g_{1}, f_{2}\right)>\lambda\right\}\right| \leq \frac{1}{\lambda^{2}} \int\left|T_{K}\left(g_{1}, f_{2}\right)\right|^{2} \leq \frac{C}{\lambda^{2}}\left\|g_{1}\right\|_{L^{2}}^{2}\left\|f_{2}\right\|_{L^{\infty}}^{2} \leq \frac{C}{\lambda},
$$

(by the Coifman-Meyer result [C-M4]), giving the fact that $T_{K}\left(f_{1}, f_{2}\right)$ is in weak $L^{1}$. Using Theorem 3 (with $\ell=1$ ), we now obtain that, for $f_{2} \in L^{r_{2}}$, $T_{K}\left(f_{1}, f_{2}\right) \in L^{s, \infty}, \frac{1}{s}=1+\frac{1}{r_{2}}, 1 \leq r_{2} \leq \infty$, which yields (b). Finally, to obtain (a), we use (b) and Remark 4 above, together with the Coifman-Meyer result [C-M4] for $1<r_{1}<\infty, r_{2}=\infty$, and $r_{1}=\infty, 1<r_{2}<\infty$.

\section{Concluding Remarks}

Remark 10. The operators $I_{\alpha, k}$, and $T_{K}$ appearing in Lemma 7 and Theorem 9 can be put in a more general form in parallel with the $(k+1)$-linear operators arising in Theorem 1. Here we define $I_{\alpha, k}$ and $T_{K}$ as

$$
\int_{\mathbb{R}^{n k}} f_{1}\left(\ell_{1}\right) f_{2}\left(\ell_{2}\right) \cdots f_{k}\left(\ell_{k}\right) K\left(x_{1}, \ldots, x_{k}\right) d x_{1} \cdots d x_{k}
$$

with $K(x)=\left|\left(x_{1}, \ldots, x_{k}\right)\right|^{-n k+\alpha}$, or $K(x)$ a Calderón-Zygmund kernel on $\mathbb{R}^{n k}$. Also

$$
\ell_{j}=\ell_{j}\left(x_{1}, \ldots, x_{k}, x\right)=\sum_{i=1}^{k} A_{i j} x_{i}+A_{k+1, j} x, 1 \leq j \leq k .
$$

We make the following assumptions analogous to (H1), (H2), (H3):

(h1) For each $j, 1 \leq j \leq k$, the matrix $A_{k+1, j}$ is invertible.

(h2) The $k n \times k n$ matrix $\left(A_{i, j}\right)_{\substack{1 \leq i \leq k \\ 1 \leq j \leq k}}$ is invertible.

Then the conclusions of Lemma 7 and Theorem 9 still hold in this more general term. This can easily be deduced from the special cases already proved. First we replace the functions $f_{1}$ by $f_{1}^{\prime}$ with $f_{j}^{\prime}\left(A_{k+1, j}^{-1}(x)\right)=f_{j}(x)$. Then we write $A_{k+1}^{-1} \ell_{j}=x-\sum_{i=1}^{k} A_{i j}^{\prime} x_{i}$ with $A_{i j}^{\prime}=-A_{k+1, j}^{-1} \cdot A_{i j}$. Finally one makes the linear change of variables $y_{j}=\sum_{i=1}^{k} A_{i j}^{\prime} x_{i}$.

\section{References}

[B-L-L] H.J. Brascamp, E.H. Lieb and J.M. Luttinger, A general rearrangement inequality for multiple integrals, J. Functional Analysis 17 (1974), 227-237.

[C-M1] R.R. Coifman and Y. Meyer, On commutators of singular integrals and bilinear singular integrals, Trans. Amer. Math. Soc. 212 (1975), 315-331.

[C-M2] _ Commutateurs d'integrales singulières et opérateurs multilinéaires, (French) Ann. Inst. Fourier (Grenoble) 28 (1978), 177-202. 
[C-M3] Au-delà des opérateurs pseudo-différentiels, Astérisque 57, Société Mathématique de France, Paris, 1978.

[C-M4] _ Non-linear harmonic analysis, operator theory and P.D.E., Beijing Lectures in Analysis, Annals of Math. Studies 112 (1986), 3-46.

[C-M5] , Ondelettes et opérateurs III, Opérateurs multilinéaires, Actualités Mathématiques, Hermann, Paris, 1991.

[G] L. Grafakos, On multilinear fractional integrals, Studia Math. 102 (1992), 49-56.

[G-K] L. Grafakos and N. Kalton, Some remarks on multilinear maps and interpolation, to appear.

[J] S. Janson, On interpolation of multilinear operators, 290-302, Springer Lecture Notes in Math. 1302, Springer-Verlag, Berlin-New York, 1988.

[L-T] M. Lacey and C. Thiele, On Calderón's conjecture, to appear, Annals of Math.

[S] E. Stein, Singular integrals and differentiability properties of functions, Princeton Mathematical Series, No. 30, Princeton University Press, Princeton, N.J., 1970.

[S-W] E. Stein and G. Weiss, Introduction to Fourier analysis on Euclidean spaces, Princeton Mathematical Series, No. 32, Princeton University Press, Princeton, N.J., 1971.

Department of Mathematics, University of Chicago, Chicago, IL 60637

E-mail address: cek@math.uchicago.edu

Department of Mathematics, Princeton University, Princeton, NJ 08544

E-mail address: stein@math.princeton.edu 